\title{
TENDENCIAS DE LA TEMPERATURA MENSUAL Y DE LOS EXTREMOS DIARIOS DURANTE EL PERÍODO 1966-2015 EN EL NORTE GRANDE CHILENO
}

\author{
Óliver MESEGUER-RUIZ ${ }^{1,2}$, Oscar CORVACHO ${ }^{1}$, Alejandro TAPIA TOSETTI ${ }^{1}$, \\ José Fabián LÓPEZ-CEPEDA ${ }^{1}$, Pablo SARRICOLEA ${ }^{2,3}$ \\ ${ }^{1}$ Departamento de Ciencias Históricas y Geográficas, Universidad de Tarapacá. \\ ${ }^{2}$ Grupo de Climatología, Universidad de Barcelona. \\ ${ }^{3}$ Departamento de Geografia, Universidad de Chile. \\ omeseguer@uta.cl, ocorvacho@uta.cl, atapia@uta.cl, jlopezc@uta.cl, psarricolea@uchilefau.cl
}

\section{RESUMEN}

Según el V informe del Intergovernmental Panel on Climate Change, el norte de Chile es uno de los territorios que más se verán afectados por los cambios en las dinámicas atmosféricas en los próximos años. Dentro de este contexto general, un pilar capital lo constituyen las temperaturas y las modificaciones que en ella se han venido registrando. Se ha estudiado el comportamiento de las temperaturas de 4 observatorios del norte de Chile. Para detectar posibles tendencias en las series, se ha aplicado el test no paramétrico de Mann-Kendall, en primer lugar, a las temperaturas medias mensuales, y, posteriormente, a 12 índices diferentes calculados a partir de los extremos diarios de temperaturas desde 1966 hasta 2015. Si bien las medias mensuales no muestran unas tendencias claras significativas en el período estudiado, coincidiendo con los estudios que señalan la observación del Global Warming Hiatus, los otros índices de los extremos, muestran tendencias significativas (90\%), que indican, principalmente, un claro aumento de las temperaturas mínimas, así como una disminución evidente del número de días anuales en los que se registran temperaturas del percentil inferior.

Palabras clave: Temperatura media, temperatura extrema, tendencia, Norte Grande, Chile

\begin{abstract}
According to the AR5 from the Intergovernmental Panel on Climate Change, northern Chile is one of the most affected region by the next years-changes in the atmospheric dynamics. Within this general context, the temperature and its modifications constitute a capital pillar. The behavior of the temperatures from 4 observatories in northern Chile have been studied. To detect possible trends in the time series, the non-parametrical Mann-Kendall test has been applied to the monthly mean temperatures in a first place, and, after that, to twelve different indices calculated according to the daily extreme temperature from 1966 to 2015. While monthly means do not show a significant trend in the studied period, coinciding with studies indicating the observation of the Global Warming Hiatus, the other extremes indices show significant (90\%) trends, indicating a clear increase in minimum temperatures and a clear decrease in the number of annual days with minimum temperatures in the lower percentile.
\end{abstract}


Key words: Mean temperature, extreme temperature, trend, Norte Grande, Chile

\section{INTRODUCCIÓN}

En la actualidad, a nivel planetario se asiste a un contexto ambiental marcado por el Cambio Climático (IPCC, 2013) que afecta a las diferentes variables atmosféricas de distintas maneras, y el marco conceptual más generalmente aceptado corresponde al calentamiento global, entendido como la tendencia al aumento de las temperaturas planetarias. El Norte Grande chileno (desde los $17^{\circ}$ hasta los $29^{\circ} \mathrm{S}$ ) es afectado por unas dinámicas atmosféricas concretas que otorgan a esta región una personalidad climática muy particular, con un carácter seco predominante por encima de todos. Las preocupaciones crecientes sobre los impactos del cambio climático relativos a las temperaturas hace que ésta sea una de las variables meteorológicas más investigadas, en parte debido a su influencia en los sistemas naturales y en las actividades humanas (IPCC, 2013). Numerosos estudios han demostrado que el aumento de las temperaturas puede acelerar e intensificar los ciclos climático e hidrológico, afectando la precipitación global en lo referente a su cantidad y a su intensidad, al régimen de los ríos, a la humedad del suelo y a los balances de evapotranspiración (Guan et al., 2015). El aumento de los valores de temperatura media a lo largo del último siglo ha sido ampliamente demostrado por numerosos estudios en diferentes regiones del mundo, tanto en una escala espacial amplia (Klok y Tank, 2009) como a escala local (Pellicone et al., 2014). En lo que refiere al Pacífico Sur, Salinger (1995) evidenció que las temperaturas máximas y mínimas diarias se han incrementado generalmente en toda el área en los últimos años del siglo pasado. Además, la ocurrencia de eventos cálidos extremos acoplados con aumentos de las temperaturas medias han ocurrido más frecuentemente en el período 1957-1996, a la vez que se ha evidenciado un descenso en el número de los eventos fríos (Plummer et al., 1999).

En adición a las temperaturas medias, los investigadores también han trabajado sobre la variabilidad de los extremos de la temperatura del aire superficial durante las últimas décadas (Guan et al., 2015). De hecho, la comunidad climática se muestra de acuerdo en el hecho que cualquier cambio en la frecuencia o intensidad de los fenómenos meteorológicos extremos podría tener consecuencias más serias y profundas en la agricultura, los ecosistemas y en la sociedad que aquellos producidos en los valores medios (Guan et al., 2015). Katz y Brown (1992) evidenciaron que el cambio climático afecta principalmente a los valores extremos, mientras que pequeños cambios en las condiciones medias puede provocar grandes cambios en la frecuencia de los extremos (Salinger y Griffiths, 2001). A nivel global se han detectado cambios relevantes en los extremos a lo largo del siglo XX. Particularmente, mientras que se ha evidenciado una tendencia clara en la mayoría de los extremos relativos a bajas temperaturas en multitud de regiones a lo largo del mundo a finales del siglo XX, no se han detectado al mismo nivel cambios significativos relativos a las altas temperaturas (Salinger y Griffiths, 2001).

Es bien conocida la importancia de la agricultura en Chile, y ésta es sensible a las fluctuaciones de las temperaturas entre un año y el siguiente. Las altas temperaturas pueden agravar las sequías e incrementar la posibilidad de incendios. Muy altas temperaturas pueden también dañar los cultivos y reducir su rendimiento. En oposición, 
las bajas temperaturas pueden provocar heladas y, dependiendo de la parte del país, fuertes tormentas de nieve; aunque las bajas temperaturas también son importantes para determinadas especies hortícolas y para el crecimiento de algunos cereales (Salinger y Griffiths, 2001). En el caso específico del Norte Grande chileno, área desértica poblada por más de un millón de habitantes con el 95\% de ellos urbanos (INE, 2010), un aumento de las temperaturas puede incidir en la disponibilidad de agua para uso doméstico, para una agricultura de oasis de gran importancia regional, y, en especial, para la industria extractiva minera localizada en la zona, principal actividad económica del país.

Sin embargo, en los últimos años se ha dado una disminución del ritmo del calentamiento cuyas causas aún están por determinar. Algunos investigadores afirman que la sensibilidad del clima podría haberse sobreestimado (Otto et al., 2013), otros apuntan a la incompleta cobertura a nivel global de las observaciones de temperatura y que este aumento de las mismas no está quedando registrado en aquellas regiones donde no hay una buena red de observatorios (Cowtan y Way, 2014), o también debido a cambios en la actividad solar y en los aerosoles estratosféricos y troposféricos que hubieran provocado un estancamiento de las tendencias de temperatura en la última década (Solomon et al., 2011).

Sin embargo, no existe otro lugar a nivel mundial en el que este receso en el calentamiento aparezca de forma tan evidente como a lo largo de la costa del norte de Chile (Vuille et al., 2015) y en sus montañas (Bennet et al., 2016), donde la temperatura aumentó significativamente durante la mayor parte del siglo $\mathrm{XX}$, pero luego empezó a decaer, resultando en un enfriamiento sostenido de hasta $-0,20^{\circ} \mathrm{C} /$ década a lo largo de los últimos 20-30 años (Falvey y Garreaud, 2009). Falvey y Garreaud (2009) atribuyeron parcialmente el enfriamiento observado a cambios en la variabilidad decadal del Pacífico y sugirieron que la intensificación proyectada del APS y la consiguiente intensificación de las corrientes de 'upwelling' de aguas frías por debajo de la termoclina ayudarían a enfriar la región en el futuro. Independientemente de este enfriamiento lejos de la costa, los glaciares andinos siguen retrocediendo (Rabatel et al., 2013). Al mismo tiempo, la temperatura a lo largo de la costa occidental del Pacífico tropical (Perú y Ecuador) alcanzó valores máximos en la segunda mitad del siglo XX (Vuille y Bradley, 2000).

Un tema común que emerge de todos estos estudios es que parecen existir amplias diferencias entre las tendencias de las temperaturas observadas entre los trópicos y las áreas fuera de ellos y entre el calentamiento de los continentes y el enfriamiento de los océanos lejos de las costas. Sigue siendo incierto si el reciente enfriamiento anómalo afecta únicamente las latitudes medias de la costa occidental de Sudamérica o si sus efectos se extienden hasta los trópicos. Estudios más antiguos no detectan este enfriamiento (Vuille y Bradley, 2000), pero esto podría reflejar también que únicamente se trabajó aquí con datos del siglo $\mathrm{XX}$, lo que no incluiría el reciente período de enfriamiento.

Los objetivos de este trabajo consistirán por lo tanto en analizar las tendencias de las temperaturas máximas y mínimas mensuales y después investigar la magnitud y la distribución espacial de los extremos de la temperatura en el Norte Grande chileno durante el período 1966-2015 utilizando 12 índices propuestos por el Expert Team on 
Climate Change Detection Indices (ETCCDI) (Zhang et al., 2011), así como arrojar luz sobre las características espaciotemporales de las variaciones de la temperatura en el norte de Chile.

\section{MÉTODOS}

Con el objetivo de detectar cambios en los extremos climáticos, es importante desarrollar una serie de índices estadísticamente robustos y que sean aplicables a la mayoría de climas mundiales. Los índices internacionalmente aceptados para las temperaturas diarias arrojan resultados que pueden ser comparados consistentemente entre diferentes países y regiones y sobrepasan las restricciones que presentan la habitual diseminación de observatorios diarios en la mayor parte de las regiones. Para poder determinar la posible existencia de tendencias temporales en las máximas y mínimas mensuales y en los índices de extremos, las series de temperaturas medias mensuales y extremas diarias se analizan, y su significación estadística vendrá determinada por el conocido test no paramétrico de Mann-Kendall (MK).

Klein Tank et al. (2002) definieron una serie de índices que, posteriormente, pasaron a ser conocidos como los índices ETCCDI y que se basaban en los índices propuestos previamente por la European Climate Assessment (ECA) (2002) para analizar tendencias en la segunda mitad del siglo XX. Estos índices fueron seleccionados para que pudieran albergar una amplia variedad de climas. Para este trabajo se aplicará una selección de 12 de los 29 índices del ETCCDI (Tabla 1).

\begin{tabular}{|c|c|c|c|}
\hline Índice & Nombre & Definición & Unidad \\
\hline TXx & $\mathrm{T}_{\max } \operatorname{Max}$ & Valor máximo mensual de la temperatura máxima diaria & ${ }^{\circ} \mathrm{C}$ \\
\hline $\mathrm{TNx}$ & $\mathrm{T}_{\min } \operatorname{Max}$ & Valor máximo mensual de la temperatura mínima diaria & ${ }^{\circ} \mathrm{C}$ \\
\hline TXn & $\mathrm{T}_{\max } \operatorname{Min}$ & Valor mínimo mensual de la temperatura máxima diaria & ${ }^{\circ} \mathrm{C}$ \\
\hline $\mathrm{TNn}$ & $\mathrm{T}_{\min } \operatorname{Min}$ & Valor mínimo mensual de la temperatura mínima diaria & ${ }^{\circ} \mathrm{C}$ \\
\hline TN10p & Noches frías & Conteo anual de temperaturas mínimas diarias $<10^{\circ}$ percentil & Días \\
\hline TX10p & Días fríos & Conteo anual de temperaturas máximas diarias $<10^{\circ}$ percentil & Días \\
\hline TN90p & $\begin{array}{l}\text { Noches } \\
\text { cálidas }\end{array}$ & Conteo anual de temperaturas mínimas diarias $>90^{\circ}$ percentil & Días \\
\hline TX90p & Días cálidos & Conteo anual de temperaturas máximas diarias $>90^{\circ}$ percentil & Días \\
\hline DTR & $\begin{array}{l}\text { Rango diario } \\
\text { de temperatura }\end{array}$ & $\begin{array}{l}\text { Diferencia media mensual entre la temperatura máxima y } \\
\text { mínima diaria }\end{array}$ & ${ }^{\circ} \mathrm{C}$ \\
\hline SU25 & $\begin{array}{l}\text { Días de } \\
\text { verano }\end{array}$ & Conteo anual de días con temperatura máxima $>25^{\circ} \mathrm{C}$ & Días \\
\hline TR20 & $\begin{array}{l}\text { Noches } \\
\text { tropicales }\end{array}$ & Conteo anual de días con temperatura mínima $>20^{\circ} \mathrm{C}$ & Días \\
\hline WSDI & $\begin{array}{l}\text { Indicador } \\
\text { de duración } \\
\text { de período } \\
\text { cálido }\end{array}$ & $\begin{array}{l}\text { Conteo anual de eventos de al menos } 6 \text { días consecutivos con } \\
\text { temperatura máxima }>90^{\circ} \text { percentil }\end{array}$ & Días \\
\hline
\end{tabular}

Tabla 1: Índices de temperatura extrema utilizados. Fuente: Extraído y modificado de Klein Tank et al. (2002). 
Algunos de estos índices (TXx. TNx, TXn y TNn) miden la temperatura diaria máxima o mínima a nivel mensual y/o anual. Otros índices permiten calcular cuántos días al año exceden umbrales específicos con valores fijos o que hacen referencia un período climático de base, como ya se ha comentado. Sin embargo, los índices que se basan en umbrales percentiles (TN10p, TX10p, TN90p, TX90p y WSDI) son preferibles a la hora de realizar comparaciones espaciales de los extremos. Esto se debe a que las muestras de distribución de temperatura pueden ser diferentes cuando se usan índices de conteo con umbrales fijos en áreas grandes. Por ejemplo, el calor continuado en climas de latitudes medias puede encontrar un buen indicador en el número de días con temperatura mínima superior a $20^{\circ} \mathrm{C}$ (SU25); por el contrario, en latitudes bajas, donde la mayor parte de las noches estivales arrojan temperaturas mínimas por encima de ese umbral, la variabilidad en el número anual de noches con temperaturas superiores a $20^{\circ} \mathrm{C}$ viene modulado por las condiciones en primavera y en otoño. Además, un índice, como el número de días de verano con máximas superiores a $25^{\circ} \mathrm{C}$ (SU25), puede indicar condiciones anormalmente cálidas en un clima templado habitual, donde la media de las máximas de verano ronda los $18^{\circ} \mathrm{C}$ (Zhang et al., 2011).

\section{RESULTADOS}

\subsection{Análisis de las temperaturas mensuales}

Los resultados de los análisis de tendencias aplicados a las series de temperatura media mensual de los 4 observatorios (nivel de significancia del 90\%) se muestran en la tabla 2 y en la figura 1. En los observatorios de Arica y Antofagasta se ha dado una tendencia al aumento de las temperaturas medias mensuales, en los primeros meses del año en ambos casos (con ritmos máximos de 0,19 y $0,21^{\circ} \mathrm{C} /$ década respectivamente).

En los meses tardo-invernales y primaverales para Antofagasta también se da un aumento significativo de las medias mensuales, llegando a $0,18^{\circ} \mathrm{C} /$ década en el mes de septiembre. En el caso de la ciudad de Iquique, las tendencias de las temperaturas medias se ha registrado a la baja, significativamente en el último trimestre anual, con valor máximo de $-0,29^{\circ} \mathrm{C} /$ década en el mes de octubre. En el observatorio de Calama no se ha registrado ninguna tendencia significativa en las medias mensuales.

\begin{tabular}{|c|c|c|c|c|c|c|c|c|c|c|c|c|}
\hline Observ. & Ene & Feb & Mar & Abr & May & Jun & Jul & Ago & Sep & Oct & Nov & Dic \\
\hline Arica & $\mathbf{0 , 1 4}$ & $\mathbf{0 , 1 5}$ & 0,09 & $\mathbf{0 , 1 9}$ & 0,17 & 0,14 & 0,13 & 0,13 & 0,07 & 0,09 & 0,10 & 0,14 \\
\hline Iquique & $-0,10$ & 0,00 & $-0,17$ & $-0,25$ & $-0,16$ & $-0,06$ & $-0,11$ & $-0,11$ & $-0,17$ & $\mathbf{- 0 , 2 9}$ & $\mathbf{- 0 , 2 0}$ & $\mathbf{- 0 , 1 8}$ \\
\hline Calama & 0,05 & 0,00 & 0,05 & 0,00 & 0,00 & 0,05 & 0,00 & 0,05 & 0,00 & 0,00 & 0,00 & $-0,09$ \\
\hline Antofagasta & $\mathbf{0 , 1 2}$ & $\mathbf{0 , 2 1}$ & $\mathbf{0 , 1 7}$ & 0,05 & 0,03 & 0,08 & 0,00 & $\mathbf{0 , 1 6}$ & $\mathbf{0 , 1 8}$ & $\mathbf{0 , 1 3}$ & 0,05 & 0,00 \\
\hline
\end{tabular}

Tabla 2: Resultados del análisis de tendencias de las temperaturas medias mensuales. Los valores significativos (90\%) aparecen en negrita. La tendencia aparece en ${ }^{\circ} \mathrm{C} /$ década. 


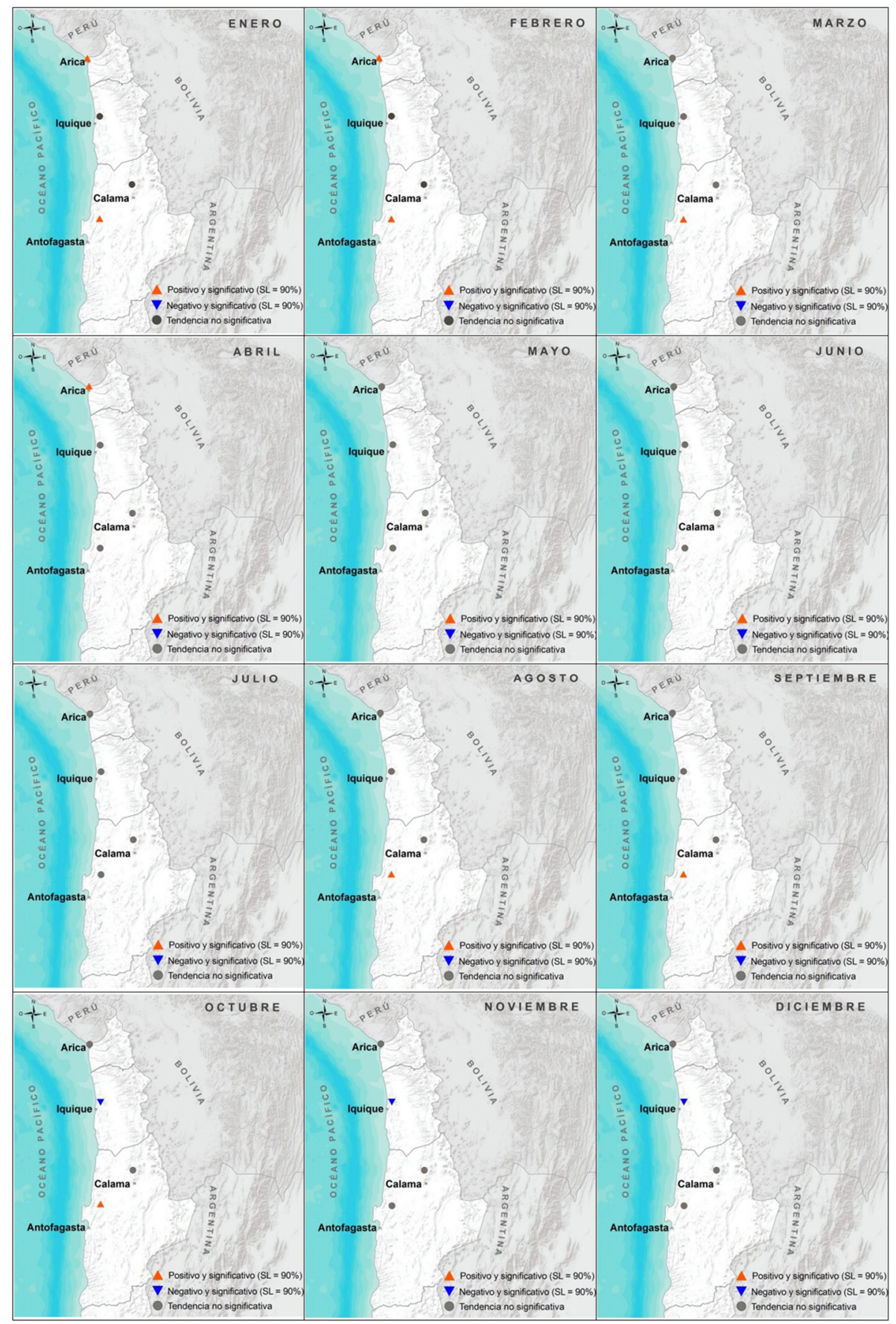

Fig. 1: Distribución espacial de las tendencias de las medias mensuales para los diferentes observatorios. Fuente: Elaboración propia. 


\subsection{Análisis de las temperaturas extremas diarias}

El análisis de las temperaturas extremas diarias en el norte de Chile revela cambios significativos (nivel de significancia del 90\%), tal y como se muestra en la tabla 3 y en la figura 2 .

\begin{tabular}{|c|c|c|c|c|c|c|}
\hline Observatorio & TXx & TNx & TXn & TNn & TN10p & TX10p \\
\hline Arica & 0,04 & $\mathbf{0 , 2 9}$ & 0,00 & $\mathbf{0 , 4 7}$ & $\mathbf{- 8 , 9 4}$ & 4,29 \\
\hline Iquique & $\mathbf{0 , 3 5}$ & $\mathbf{0 , 3 8}$ & $-0,08$ & $\mathbf{0 , 3 8}$ & $\mathbf{- 3 , 3 3}$ & 3,14 \\
\hline Calama & $-0,14$ & 0,12 & $\mathbf{- 0 , 1 5}$ & $\mathbf{0 , 2 6}$ & $\mathbf{- 5 , 0 0}$ & $\mathbf{5 , 0 0}$ \\
\hline Antofagasta & 0,18 & $\mathbf{0 , 2 9}$ & 0,13 & $\mathbf{0 , 2 3}$ & $-1,67$ & 0,35 \\
\hline Observatorio & TN90p & TX90p & DTR & SU25 & TR20 & WSDI \\
\hline Arica & $\mathbf{7 , 6 7}$ & $\mathbf{- 6 , 5 6}$ & $\mathbf{- 0 , 5 1}$ & $\mathbf{- 8 , 7 5}$ & $\mathbf{8 , 0 0}$ & 0,00 \\
\hline Iquique & $\mathbf{7 , 6 8}$ & $\mathbf{5 , 0 0}$ & $\mathbf{- 0 , 1 7}$ & $\mathbf{6 , 3 2}$ & $\mathbf{1 , 6 7}$ & $\mathbf{0 , 3 4}$ \\
\hline Calama & $\mathbf{3 , 9 1}$ & $\mathbf{- 3 , 0 2}$ & $\mathbf{- 0 , 3 4}$ & $\mathbf{- 1 , 5 1}$ & 0,00 & 0,00 \\
\hline Antofagasta & $\mathbf{4 , 6 9}$ & $-0,26$ & $\mathbf{- 0 , 1 4}$ & 0,00 & 0,00 & 0,00 \\
\hline
\end{tabular}

Tabla 3: Resultados del análisis de tendencias de los índices de temperaturas diarias extremas. Los valores significativos (90\%) aparecen en negrita. La tendencia aparece en ${ }^{\circ} \mathrm{C} /$ década o días/década, según índice (ver tabla 1). Fuente: Elaboración propia.

Un comportamiento significativo del índice TXx únicamente se registra en Iquique, con un aumento de $0,35^{\circ} \mathrm{C} /$ década, mientras que el TXn solo es representativo en Calama, donde este índice presenta una tendencia de $-0,15^{\circ} \mathrm{C} /$ década. Las temperaturas mínimas, por su parte, presentan un comportamiento mucho más al alza, ya que muestran un aumento significativo de las más elevadas en las localidades costeras y en todos los observatorios en el comportamiento de las mínimas más elevadas. En la misma línea han evolucionado los índices TN10p y TN90p, que presentan una diminución de hasta 8,94 días por década de días con temperaturas mínimas en el percentil $10 \%$ inferior, mientras que las que se encuentran el 10\% superior aumentan en todos los observatorios considerados. El comportamiento de las temperaturas máximas no es tan evidente, disminuyendo incluso en dos casos.

Esto ha provocado que las diferencias entre las máximas y las mínimas diarias se hayan reducido de manera significativa en todos los casos, como muestra el índice DTR. Por otro lado, el índice SU25 muestra resultados diversos, con una disminución significativa en Arica de casi 9 días por década, un aumento de más de 6 en Iquique, una nueva disminución de 1,5 en Calama, y sin tendencia en Antofagasta. Las noches tropicales, representadas por el índice TR20, han aumentado significativamente en Arica y en Iquique, 8 y 1,67 días/década respectivamente. Más al sur, no hay cambios significativos. Las rachas de días cálidos, WSDI, únicamente aumentan en la ciudad de Iquique, y lo hacen de manera suave (0,34 días/década). 


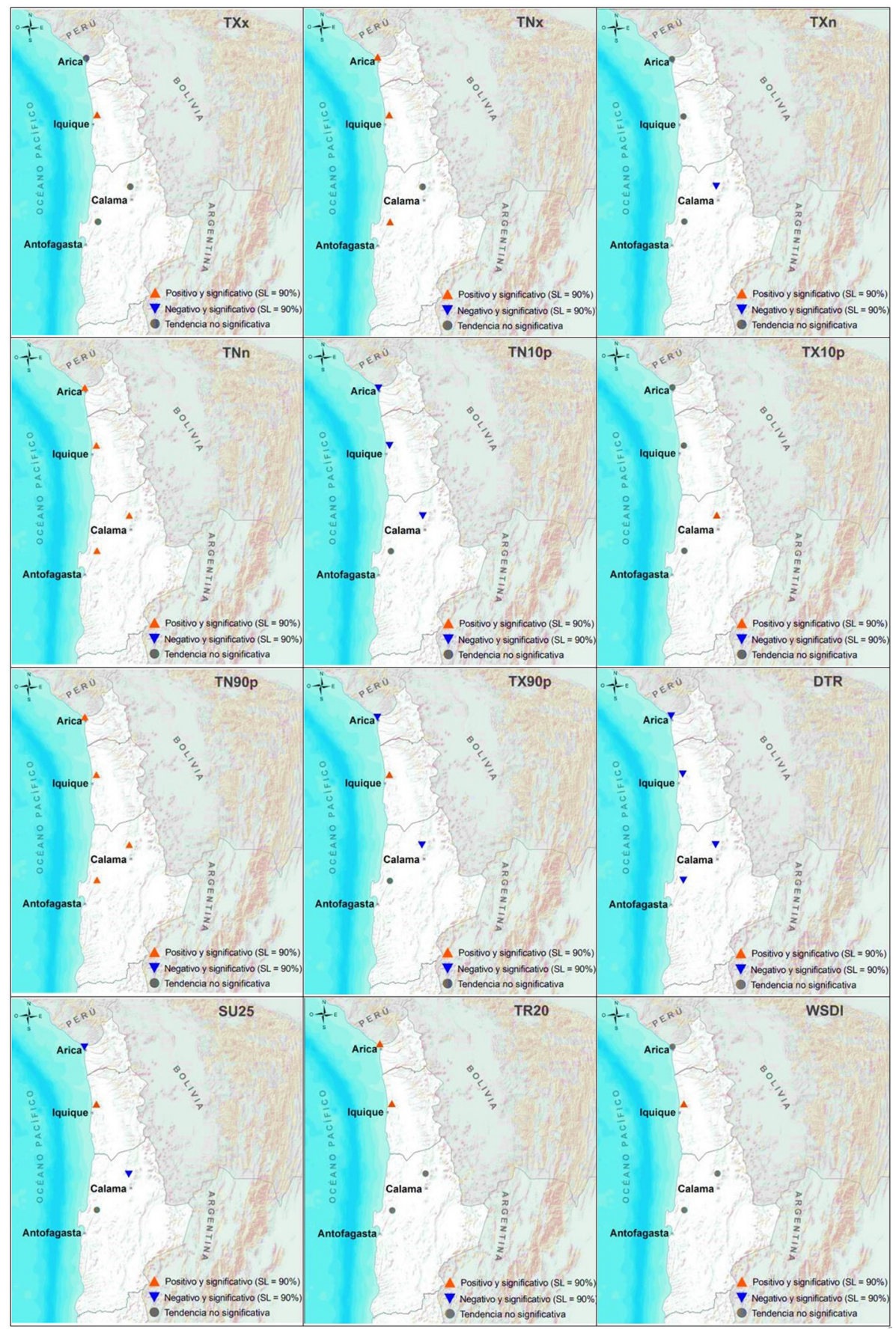

Fig. 2: Distribución espacial de las tendencias de los indices de temperaturas diarias extremas para los diferentes observatorios. Fuente: Elaboración propia. 


\section{DISCUSIÓN Y CONCLUSIONES}

Si bien no hay una tendencia muy clara en las medias mensuales, sí que se registra que las temperaturas han experimentado un ascenso en los observatorios de la costa, no así en el del interior, coincidiendo parcialmente con las proyecciones del AR5 (IPCC, 2013). El observatorio de Calama, en el interior, no presenta tendencias significativas en ninguna media mensual, lo que evidenciaría el Global Warming Hiatus en las regiones de montaña, coincidiendo con los resultados de Bennet et al. (2016), pero no plenamente con Vuille et al. (2015). Los resultados obtenidos no coinciden con los que presentan Falvey y Garreaud (2009), ya que los índices de extremos muestran un claro aumento de las temperaturas mínimas, y únicamente se evidencia una disminución de las temperaturas medias para el mes de octubre en el observatorio de Iquique.

En definitiva, las principales evidencias del aumento de las temperaturas en el Norte Grande Chileno quedarían registradas en el comportamiento de las temperaturas mínimas, habiendo registrado un aumento significativo en el período estudiado, mientras que las temperaturas máximas no han aumentado de forma generalizada.

\section{AGRADECIMIENTOS}

Los autores quieren agradecer a la Dirección Meteorológica de Chile (DMC) por la cesión de los datos. También el apoyo institucional del Convenio de Desempeño UTA-MINEDUC, del proyecto UTA-Mayor 5744-16, de los proyectos FONDECYT 11130629 y 1150701, y del Grupo de Climatología (SGR 2014/300).

\section{REFERENCIAS}

Bennet, M., New, M., Marino, J., \& Sillero-Zubiri, C. (2016). Climate complexity in the Central Andes: A study case on empirically-based local variations in the dry Puna. Journal of Arid Environments, 128, 40-49.

Cowtan, K., \& Way, R.G. (2014). Coverage bias in the HadCRUT4 temperature series and its impact on recent temperature trends. Quarterly Journal of the Royal Meteorological Society, 140, 1935-1944.

Falvey, M., \& Garreaud, R.D. (2009). Regional cooling in a warming world: Recent temperature trends in the southeast Pacific and along the west coast of subtropical South America (1979-2006). Journal of Geophysical Research, 114, D04102.

Guan, Y., Zhang, X., Zheng, F., \& Wang, B. (2015). Trends and variability of daily temperature extremes during 1960-2012 in the Yangtze River Basin, China. Global and Planetary Change, 124, 79-94.

Instituto Nacional de Estadísticas (2010). Compendio Estadístico. Santiago: Estadísticas Demográficas.

IPCC (2013). Climatic Change 2013: The Physical Science Basis. Working Group I Contribution to the Fifth Assessment Report of the Intergovernmental Panel on Climate Change. New York: Cambridge University Press.

Katz, R.W., \& Brown, B.G. (1992). Extreme events in a changing climate: variability is more important than averages. Climate Change, 21, 289-302. 
Klein Tank, A.M.G., Wijngaard, J.B., Koennen, G.P., Boehm, R., Demaree, G., Gocheva, A., Mileta, M., Pashiardis, S., Hejkrlik, L., \& Kern-Hansen, C. (2002). Daily surface air temperature and precipitation dataset 1901-1999 for European Climate Assessment (ECA). International Journal of Climatology, 22, 1441-1453.

Klok, E.J., \& Tank, A. (2009). Updated and extended European dataset of daily climate observations. International Journal of Climatology, 29, 1182-1191.

Otto, A., Otto, F.E.L., Boucher, O., Church, J., Hegerl, G., Forster, P.M., Gillett, N.P., Gregory, J., Johnson, G.C., Knutti, R., Lewis, N., Lohmann, U., Marotzke, J., Myhre, G., Shindell, D., Stevens, B., \& Allen, M.R., (2013). Energy budget constraints on climate response. Nature Geosciences, 6, 415-416.

Pellicone, G., Caloiero, T., Coletta, V., \& Veltri, A. (2014). Phytoclimatic map of Calabria (southern Italy). Journal of Maps, 10, 109-113.

Plummer, N., Salinger, M.J., Nicholls, N., Suppiah, R., Hennessy, K.J., Leighton, R.M., Trewin, B., Page, C.M., \& Lough, J.M. (1999). Changes in climate extremes over the Australian region and New Zealand during the twentieth century. Climate Change, 42, 183-202.

Rabatel, A., Francou, B., Soruco, A., Gomez, J., Cáceres, B., Ceballos, J.L.,... \& Wagnon, P. (2013). Current state of glaciers in the tropical Andes: A multi-century perspective on glacier evolution and climate change. Cryosphere, 7, 81-102.

Salinger, M.J. (1995). Southwest Pacific temperatures: trends in maximum and minimum temperatures. Atmospheric Research, 37, 87-99.

Salinger, M.J., \& Griffiths, G.M. (2001). Trends in New Zealand daily temperature and rainfall extremes. International Journal of Climatology, 21, 1437-1452.

Solomon, S., Daniel, J.S., Neely, R.R., Vernier, J.P., Dutton, E.G., \& Thomason, L.W. (2011). The persistently variable "background" stratospheric aerosol layer and global climate change. Science, 333(6044), 866-870.

Vuille, M., \& Bradley, R.S. (2000). Mean annual temperature trends and their vertical structure in the tropical Andes. Geophysical Research Letters, 27, 3885-3888.

Vuille, M., Franquist, E., Garreaud, R., Lavado Casimiro, W.S., \& Cáceres, B. (2015). Impact of the globalwarming hiatus on Andean temperature. Journal of Geophysical Research: Atmospheres, 120, doi: http://dx.doi.org/10.1002/2015JD023126

Zhang, X., Alexander, L., Hegerl, G.C., Jones, P., Tank, A.K., Peterson, T.C., Trewin, B., \& Zwiers, F.W. (2011). Indices for monitoring changes in extremes based on daily temperature and precipitation data. WIREs Climate Change, 2, 851-870. 